IA $U$ Colloquium 164: Radio Emission from Galactic and Extragalactic Compact Sources

ASP Conference Series, Vol. 144, 1998

J. A. Zensus, G. B. Taylor, \& J. M. Wrobel (eds.)

\title{
A VSOP Survey Program Pre-launch Continuum Survey
}

\author{
P. G. Edwards \& E. B. Fomalont ${ }^{1}$ \\ ISAS, 3-1-1 Yoshinodai, Sagamihara, Kanagawa 229, Japan
}

\begin{abstract}
The VSOP Survey Program will observe a complete survey of flat-spectrum sources at $5 \mathrm{GHz}$. In order to determine which of the sample sources are strong and small enough to be detected with VSOP, a pre-launch survey of 377 continuum sources north of $\delta>-43^{\circ}$ was conducted at $5 \mathrm{GHz}$ with the VLBA over 24 hours in June 1996. About 250 of the sources had correlated flux densities $\geq 0.4 \mathrm{Jy}$ between $\mathrm{MK}-\mathrm{SC}$ and have been included in the VSOP survey sample.
\end{abstract}

\section{The VSOP Survey Program}

The HALCA satellite was launched by ISAS on 12 February 1997. Observations with the HALCA satellite and the world's ground radio telescopes form the VLBI Space Observatory Programme (VSOP). Approximately $25 \%$ of the inorbit time will be devoted to mission-led Survey Program of active galactic nuclei and water vapor masers: see the VSOP Announcement of Opportunity (obtainable via http://www.vsop.isas.ac.jp) for more details.

\section{The VLBA Pre-Launch Survey}

On 1996 June $5 \& 6$ the VLBA was used for 24 hours in order to observe most of the sources in the VSOP survey source list at $5 \mathrm{GHz}$. The primary purpose of the observations were to determine if the correlated flux density of a source was sufficiently large to be included in the VSOP survey observations.

Sources with a flux density at $5 \mathrm{GHz}>1.0 \mathrm{Jy}$, a spectral index $>-0.5$, and a galactic latitude $>10^{\circ}$ were selected from the GB and PMN $5 \mathrm{GHz}$ surveys and supplemented by the addition of all sources with $S_{5 \mathrm{GHz}}^{\text {Total }}>5.0 \mathrm{Jy}$. Single 150 second snapshots were taken of 377 extragalactic sources meeting these criteria. The classification of sources by NED (the NASA/IPAC Extragalactic Database) is indicated in Table 1. The 'Other' class consisted of 22 Radio Sources and 4 Visible Sources.

Table 1. Source classification and detection statistics. The detection limit is $0.1 \mathrm{Jy}$.

\begin{tabular}{lccc}
\hline \hline Source type & Number & $\begin{array}{c}\text { Detected on } \\
\text { shortest baselines }\end{array}$ & $\begin{array}{c}\text { Detected on } \\
\text { longest baselines }\end{array}$ \\
\hline QSOs & 235 & $\mathbf{2 3 2}$ & 230 \\
BL Lacs & 51 & 51 & 50 \\
Galaxies & 65 & 50 & 46 \\
Other & 26 & 19 & 18 \\
\hline \hline
\end{tabular}

${ }^{1}$ Permanent address: National Radio Astronomy Observatory, Charlottesville, VA 22903, USA 
The data were correlated at Socorro and calibrated and edited using the AIPS system at ISAS. Flux density calibration was done using J1522-2730 with an assumed flux density of $2.28 \mathrm{Jy}$.

\section{Results}

The correlated flux as a function of $(u, v)$ distance is plotted in Figure 1 for two representative sources. J0241-0815 shows a steady decline in correlated flux with $(u, v)$ distance and, as the correlated flux on the longest baseline is $<0.4 \mathrm{Jy}$, it is not included in the VSOP survey sample. J0217+0144 is unresolved on long baselines and is a good strong compact source for space VLBI observations.

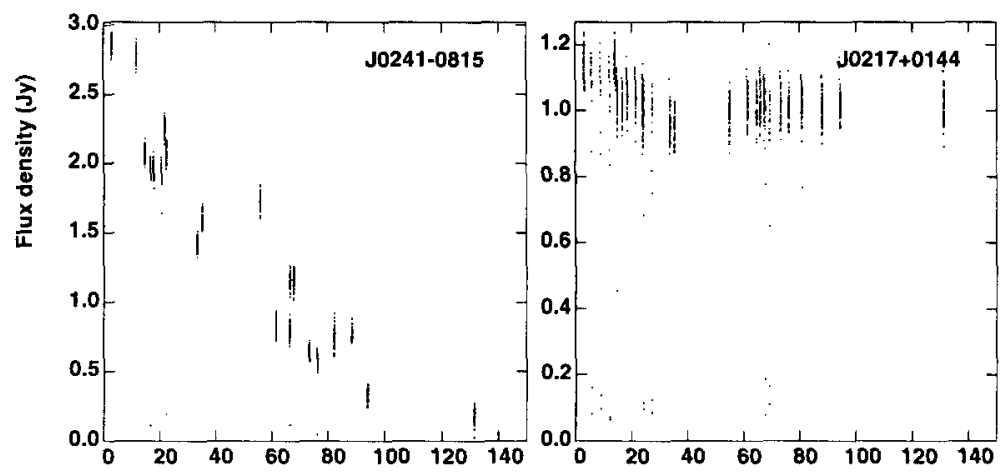

Figure 1. Flux density versus $(u, v)$ distance in $\mathrm{M} \lambda$ for two representative sources.

Plots like these were made for all sources and used to determine values of $S_{5 G H z}^{5 M \lambda}$ and $S_{5 G H z}^{120 M \lambda}$, corresponding to the shortest and longest baselines. These values are available from the VSOP WWW site.

Measurements of the total $5 \mathrm{GHz}$ flux density over the last $\sim 10$ years are available for almost all of the sources. About $\sim 40 \%$ of the sources had values of $S^{5 M \lambda}$ at least $10 \%$ greater than the previously measured total flux density, indicating that the sources were brighter in June 1996 than at the earlier epoch. Thus, variability over a decade is typical of this $5 \mathrm{GHz}$ sample.

The plots of the visibility amplitude versus baseline length have been used to determine which sources will be included in the VSOP continuum survey list. Approximately $68 \%$ of sources in the pre-launch survey had $S_{5}^{120 M \lambda} \geq 0.4$ Jy, making them good targets. In total $78 \%$ had $S_{5}^{120 M \lambda} \geq 0.3 \mathrm{Jy}$. The Survey Program observing strategy is to observe preferentially the stronger sources first, so that at all stages an almost complete flux-limited sample has been observed.

Acknowledgments. The use of the NASA/IPAC Extragalactic Database (NED) which is operated by the Jet Propulsion Laboratory, California Institute of Technology, under contract with the National Aeronautics and Space Administration, is gratefully acknowledged. The National Radio Astronomy Observatory is a facility of the National Science Foundation, operated under a cooperative agreement by Associated Universities, Inc. 